\title{
Catastrophe and Hysteresis by the Emerging of Soliton-Like Solutions in a Nerve Model
}

\author{
Fernando Ongay Larios, ${ }^{1}$ Nikolay P. Tretyakov, ${ }^{2}$ and Maximo A. Agüero ${ }^{1}$ \\ ${ }^{1}$ Faculty of Sciences, Autonomous University of the State of Mexico, 50000 Toluca, Mexico \\ ${ }^{2}$ Department of Applied Mathematics, Russian State Social University, Moscow 129226, Russia \\ Correspondence should be addressed to Fernando Ongay Larios; fernando_ongay@yahoo.com.mx
}

Received 15 June 2014; Revised 6 September 2014; Accepted 13 September 2014; Published 7 October 2014

Academic Editor: Mitsuhiro Ohta

Copyright (C) 2014 Fernando Ongay Larios et al. This is an open access article distributed under the Creative Commons Attribution License, which permits unrestricted use, distribution, and reproduction in any medium, provided the original work is properly cited.

\begin{abstract}
The nonlinear problem of traveling nerve pulses showing the unexpected process of hysteresis and catastrophe is studied. The analysis was done for the case of one-dimensional nerve pulse propagation. Of particular interest is the distinctive tendency of the pulse nerve model to conserve its behavior in the absence of the stimulus that generated it. The hysteresis and catastrophe appear in certain parametric region determined by the evolution of bubble and pedestal like solitons. By reformulating the governing equations with a standard boundary conditions method, we derive a system of nonlinear algebraic equations for critical points. Our approach provides opportunities to explore the nonlinear features of wave patterns with hysteresis.
\end{abstract}

\section{Introduction}

As is well known, one of the fundamental problems in physics applied to natural biological processes is to investigate the ways the nature transports information and energy between two or more points of living organism. On nerve fibers, for example, there exist several mathematical descriptions. The remarkable one was done by Hodgkin and Huxley $(\mathrm{HH})$ in the 50s [1]. By using this model it has been established that the dynamic of ionic currents through voltage channels is responsible for the change of the membrane potential in nerve tissues. That means the membrane contains proteins with specific behaviors of selectivity with respect to the conduction of sodium and potassium ions though the membrane. Later, Hodgkin and Huxley system was developed independently by many authors and specifically the work of Fitzhugh and Nagumo [2,3] suggested, as analogous neuronal, a nonlinear electrical circuit, controlled by an equation system also similar to those of Van Der Pol currents. Further the model was extended by modeling the nerve pulse as collective excitations from the point of view of dynamical systems; see, for example, [4].

After this successful beginning several other works concerning specifically soliton-like structures in the HH model have been done. Indeed, for example, Katz in [5] proposed the existence of traveling soliton-like pulse in this model. Muratov in his paper [6] obtained solitary waves for nerve pulses with velocities that are in certain agreement with experimental results. However, some further investigations lead to observing the dissipation of heat [7] that should have considerable distort at large distance and time the proper behavior of nerve cells.

It is suggested to say that, despite those very important achievements on the $\mathrm{HH}$ model, there is still a current problem concerning the heat releasing during the evolution of electrical nerve signals along the axons. This problem could be discarded if nature could choose the way for transmitting information and energy as evolving processes with or without little amount of heat liberation, that is, like an adiabatic process.

On the other hand, there are relevant works which report unusual behavior of soliton emergency due to hysteric processes. For example, $\mathrm{Wu}$ and coworkers [8] report the experimental observation of the generation of dark solitons inherently hysterical, through self-modulational instability moving the operating point frequency across a selected dipole gap in the dipole-exchange spin wave mode dispersion response. Higher powers lead to further changes in the power frequency 
spectra, such as comb compression and chaos. In the work of Rosanov et al. [9] the hysteresis of the velocity of switching waves (fronts) in the bistable regimes has been revealed and discrete dissipative solitons have been found.

The principal argument taken further is considering the neural activity as related to mechanical and thermodynamical properties in the axon. However, the idea of the neural signal as a mechanical wave was proposed early in 1912 by Wilke, University of Heidelberg, Germany [10]. Recently Heinburg and coworkers [11-13] considerably improved the model and better answered the question considering the wave nerve as a density pulse along the axons. From the scientific medical point of view Barz et al. [14] also supported the idea of thermomechanical responsibility of the nerve pulse propagation and appearance.

The aim of this study is to take into consideration the Heinburg model [11] and the results of our recent works [15] directed towards the description of the dark and soliton on background like traveling wave solutions, in such a way to show the emergence of catastrophe and hysteresis as fundamental properties of these solitons.

This paper is organized as follows. Section 2 discusses the setting of the current problem concerning the mechanical approach along with weak formulation in order to obtain appropriated traveling solutions. In Section 3 we discuss the algebraic analytic conditions for the potential solving the differential equation that governs the evolution of nontopological bubble and pedestal solitons. Section 4 is devoted to fundamental qualitative numerical and analytical studio related to appearance of catastrophe and hysteresis in the model. The qualitative nature of equation solutions depends on equation parameters. Section 5 presents a brief summary and discussion.

\section{Setting the Problem}

A mathematical model representing the propagation of nerve waves along an axon by considering the neural signals as propagating density pulses has been formulated. For more details on this hypothesis see $[11,16]$. An important justification for the assumption of an electromechanical process is the experimental observation of reversible heat changes in phase with the action potential and a zero net heat release during the action potential. Subsequently, this model was further studied by taking into consideration the trivial and condensate boundary conditions $[15,17]$. Thus, the equation of motion of density waves along the axon can be represented by

$$
\frac{\partial^{2} u}{\partial t^{2}}=\frac{\partial}{\partial x}\left\{\left(c_{0}^{2}+p u+q u^{2}\right) \frac{\partial u}{\partial x}\right\}-h \frac{\partial^{4} u}{\partial x^{4}} .
$$

With $u(x, t)=\rho^{A}-\rho_{0}^{A}$ representing the change of density with respect to the background density $\rho_{0}^{A}$ at physiological condition slightly above melting transition.

In this paper we will consider the nontrivial boundary condition; that is, far from the excited zone along the axon, the difference density $U$ remains constant; that is, it is not completely equal to zero.
2.1. Weak Formulation. Before the application of the boundary condition we slightly modify (1) bearing in mind the traveling wave solution with the independent variable $z=$ $x-v t$. By integrating (1) it can be transformed to the following one:

$$
h \frac{d^{2} u}{d z^{2}}=\left(c_{0}^{2}-v^{2}\right) u+\frac{1}{2} p U^{2}+\frac{1}{3} q U^{3}+C_{1} .
$$

Here $C_{1}$ is a constant of the integration. After subsequent integration one can obtain the next equation

$$
h\left(\frac{d u}{d z}\right)^{2}=C u+\left(c_{0}^{2}-v^{2}\right) u^{2}+\alpha u^{3}+\beta u^{4}+V_{0}
$$

with $\alpha=p / 3, \beta=q / 6, C=2 C_{1}$ and $p$ and $q$ being the parameters that appear in (1). The parameters $C, V_{0}$ are the constants of integration for the first and second integration correspondingly. For (3) to make sense in terms of distribution it is enough that $u \in H_{\mathrm{loc}}^{1}(R)$.

Now, let us study (3) by keeping in mind first the nontrivial or the condensate boundary condition; that means, at long distances from the main excited zone of the axon, the perturbation pulse does not vanish while its first derivative tends to zero. Thus, the unknown function $u(z)$ in the distributional sense satisfies

$$
\text { if } z \longrightarrow \infty \text { then } u \longrightarrow U_{0} \quad \frac{d u}{d z} \longrightarrow 0 \text {. }
$$

By applying this restriction, for the constants of integration $U_{0}$ and $V_{0}$ we obtained

$$
V_{0}=U_{0}\left[\left(v^{2}-c_{0}^{2}\right) U_{0}-\alpha U_{0}^{2}-\beta U_{0}^{3}-C\right] .
$$

As it can be easily seen this constant of integration depends on the background value of the difference density $U_{0}$ which far from the excited zone will remain unperturbed.

In second order equation (3) we will assume $\beta>0$ and we reparametrize the trajectories by using $s=\sqrt{\beta / h} z$ in order to obtain the dimensionless equation

$$
(\dot{u})^{2}-\left(C u+\delta u^{2}+\alpha u^{3}+u^{4}\right)=V_{0}
$$

with $\delta=\left(c_{0}^{2}-v^{2}\right) / \beta$. Equation (3) can be represented by a canonical structure for conservative systems

$$
\dot{u}_{1}=\frac{\partial H}{\partial u_{2}}, \quad \dot{u}_{2}=-\frac{\partial H}{\partial u_{1}}
$$

with $\dot{u}=\partial u / \partial s$ and the Hamiltonian

$$
H\left(u_{1}, u_{2}\right)=u_{2}^{2}-\left(C u_{1}+\delta u_{1}^{2}+\alpha u_{1}^{3}+u_{1}^{4}\right)
$$

that corresponds to the energy level

$$
H\left(u_{1}, u_{2}\right)=V_{0}
$$

being $V_{0}$ the constant of integration. Then, we rewrite boundary conditions (4) in the following manner:

$$
\lim _{s \rightarrow \pm \infty}\left(u_{1}(s), u_{2}(s)\right)=\left(U_{0}, 0\right) .
$$


One possible solution of this system is the equilibrium solution $\left(U_{0}, 0\right)$, that is, the fixed point as a critical point of $H$. Conditions (10) indicate the existence of a homoclinic trajectory that joins $\left(U_{0}, 0\right)$ with itself. In this case this critical point will be a saddle point, and the phase trajectory will surround a local minimum of the potential.

Let us assume that $\left(U_{0}, 0\right)$ is a fixed point of the system; hence $U_{0}$ must be a critical point of the potential function

$$
V\left(u_{1}\right)=-\left(C u_{1}+\delta u_{1}^{2}+\alpha u_{1}^{3}+u_{1}^{4}\right)
$$

with $V^{\prime}\left(U_{0}\right)=0$. If $\left(U_{0}, 0\right)$ is a saddle point with trajectories that surround a local minimum, then the potential $V$ has a local minimum and reaches a local maximum in $U_{0}$.

\section{Algebraic-Analytical Conditions for the Potential $V$}

Assuming $\alpha$ and $v$ (or equivalently $\delta$ ) fixed, the conditions pointed out at the end of the last section could be satisfied for a suitable interval of the parameter value $C$ as we will see in shortly. In the following paragraphs we will make an algebraic-analytical survey of the function

$$
f(x)=x^{4}+\alpha x^{3}+\delta x^{2}+C x,
$$

suggested by (11), in order to determine the desired conditions of the potential

$$
V(u)=-f(u)
$$

Since $f(x)$ is a degree 4-polynomial, the desired conditions imply that it will show three critical points being two relative minima and one relative maximum. Hence the derivative $f^{\prime}$ has three roots. The derivative is the vertical translation of a degree 3-polynomial,

$$
f^{\prime}(x)=g(x)+C,
$$

with

$$
g(x)=4 x^{3}+3 \alpha x^{2}+2 \delta x=x\left(4 x^{2}+3 \alpha x+2 \delta\right) .
$$

Obviously $f^{\prime}$ should have only three roots if its graph is $\mathrm{N}$ shaped or that is the same if the graph of $g$ is $\mathrm{N}$ shaped. By deriving $g$, one found that the necessary and sufficient condition for getting this is

$$
3 \alpha^{2}>8 \delta
$$

If this condition is satisfied, the function $g$ reaches a local maximum at

$$
x_{1}=-\frac{\alpha}{4}-\frac{\sqrt{9 \alpha^{2}-24 \delta}}{12}
$$

and a local minimum at

$$
x_{2}=-\frac{\alpha}{4}+\frac{\sqrt{9 \alpha^{2}-24 \delta}}{12} .
$$

It follows that $f^{\prime}$ has three real roots if and only if

$$
C_{\min }(\alpha, \delta)=-g\left(x_{1}\right)<C<-g\left(x_{2}\right)=C_{\max }(\alpha, \delta) .
$$

Under these conditions, the critical points of $f$ are formed by three real solutions of the next cubic equation

$$
C=-g(x)=-x\left(4 x^{2}+3 \alpha x+2 \delta\right)
$$

being those a minimum, a maximum, and a minimum.

Finally, the graph of $f$ has a vertical axis of symmetry; that is, the minima are at the same level if

$$
C=-g\left(\frac{x_{1}+x_{2}}{2}\right)=-g\left(-\frac{\alpha}{4}\right)
$$

that is, when the parameters of the motion equation satisfy the following relation:

$$
C=C^{*}(\alpha, \delta)=\frac{\alpha}{2}\left(\delta-\frac{\alpha^{2}}{4}\right) .
$$

3.1. Bubble and Pedestal Solutions. In this part we will briefly recall the explicit nontopological (bubble and pedestal) solutions of (3) that were reported early in $[15,17]$. Let us consider that the parameter $C$ of $(21)$ is delimited by $-g\left(x_{1}\right)<C<$ $-g\left(x_{2}\right)$ and let $V_{0}$ be chosen in such a manner that the lower maximum of

$$
\widetilde{U}(u)=-f(u)-V_{0}
$$

is tangent to the horizontal axis (axis $u$ ) at the point $U_{0}$ with $U_{0}$ being a solution of $(20)$ and $V_{0}=-f\left(U_{0}\right)$. Then, for some constants $m$ and $n$ the value of $\widetilde{U}(u)$ can be obtained from the next equation

$$
\widetilde{U}(u)=-\left(u-U_{0}\right)^{2}\left(\left(u-U_{0}\right)^{2}+m\left(u-U_{0}\right)+n\right),
$$

with the trait that the polynomial

$$
h(u)=\left(u-U_{0}\right)^{2}+m\left(u-U_{0}\right)+n
$$

has two distinct real roots of $\widetilde{U}$, both different to $U_{0}$ (see the last figure). Then necessarily it is needed that $m^{2}-4 n>0$. Since $U_{0}$ is not situated between the two roots of $h$, we have

$$
n=h\left(U_{0}\right)>0
$$

(between those roots $h(u)<0$, outside of those roots $h(u)>$ $0)$. With these restrictions in mind, we can rewrite (3) as

$$
(\dot{u})^{2}=\left(u-U_{0}\right)^{2}\left(\left(u-u_{0}\right)^{2}+m\left(u-U_{0}\right)+n\right) .
$$

Inserting $r=u-U_{0}$ into the last equation we obtain

$$
\dot{r}=r \sqrt{r^{2}+m r+n}
$$

or, equivalently,

$$
s-s_{0}=\int_{r_{0}}^{r} \frac{d r}{r \sqrt{r^{2}+m r+n}}
$$


with $2 r_{0}<-m-\sqrt{m^{2}-4 n}$ or $2 r_{0}>-m+\sqrt{m^{2}-4 n}$, which can be integrated by standard method as

$$
\int \frac{d r}{r \sqrt{r^{2}+m r+n}}=-\frac{1}{\sqrt{n}} \cosh ^{-1}\left(\frac{2 n+m r}{ \pm \sqrt{m^{2}-4 n r}}\right) .
$$

From here, the proper solution is obtained:

$$
u(s)=\frac{2 n}{ \pm \sqrt{m^{2}-4 n} \cosh (A-\sqrt{n} s)-m}+U_{0}
$$

with $A$ being some constant of integration.

The solution (31) embraces both two types of solitons. Indeed, by taking appropriated values and considering the singularities in the parametric space, one can retrieve the bubble or the pedestal nontopological solitons; see Figures 1 and 2.

\section{Qualitative Analysis}

In this section, we will perform a qualitative analysis of parametric family of the following system of equations:

$$
\begin{gathered}
\dot{u}_{1}=\frac{\partial H}{\partial u_{2}}\left(u_{1}, u_{2}, \alpha, \delta, C\right)=u_{2}, \\
\dot{u}_{2}=-\frac{\partial H}{\partial u_{1}}\left(u_{1}, u_{2}, \alpha, \delta, C\right),
\end{gathered}
$$

with $H$ being the Hamiltonian defined by (8). This system is equivalent to second order nonlinear equation (2). The potential piece of this Hamiltonian takes the form

$$
V(u, \alpha, \delta, C)=-f(u, \alpha, \delta, C) .
$$

Equation (2) or the equivalent conservative system (32) depends essentially on the parameters $\alpha, \delta$, and $C$; the parameter $\delta$ depends on the velocity $v$ of the traveling wave, which is a solution of the original differential equation of the model. The value $\delta=0$ corresponds to traveling waves with sound velocity $c_{0}$, while $\delta<0$ corresponds to supersonic solutions and $\delta>0$ corresponds to subsonic solutions. There is an upper bound for $\delta$ when $v=0: \delta \leq c_{0}^{2} / \beta$; theoretically there is also a lower bound for the velocity $v$ imposed by the light velocity, but since this value is much greater than $c_{0}$ we can assume that $\delta$ is not lower-bounded. The parameter $C$ is a constant of integration determined by initial conditions or by some other conditions.

The inequalities (19) define a region in the parameter space $(\alpha, \delta, C)$ that is contained in one of the two regions determined by the parabolic cylinder $3 \alpha^{2}=8 \delta$ in accordance with the inequality (16). We will call the region (19) as the region of admissible parameters for the existence of desired solutions (cf. the second section). Inside the admissible region, system (32) has three equilibria (except for a measure zero set) along the horizontal axis $u_{1}$ : a center between two saddle points.

Figure 3 is a slide of the admissible region with $\alpha$ being constant. The vertical straight line on the right is the intersection of the parabolic cylinder (16) with the plane $\alpha=$ cte.

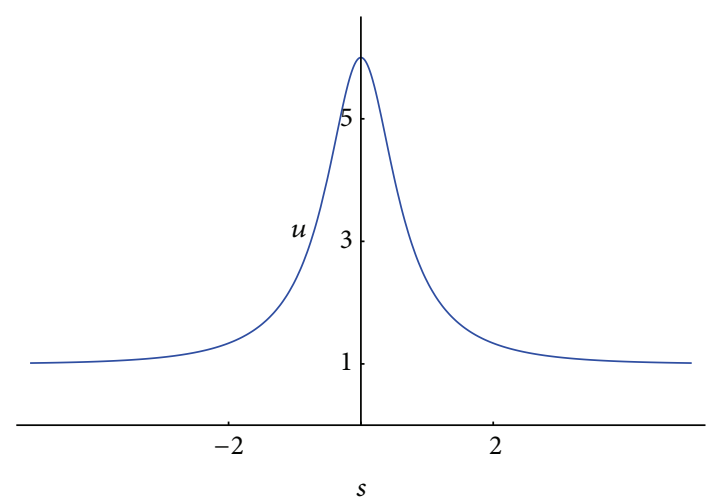

FIgURE 1: Pedestal or soliton on background.

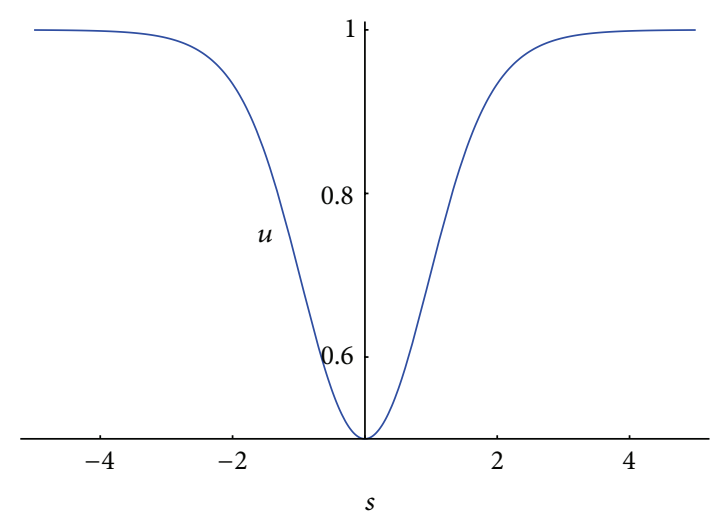

FIGURE 2: Bubble or dark like soliton.

4.1. Bifurcations. Let us consider that the parameter $C$ satisfies the next inequality

$$
C_{\min }(\alpha, \delta)<C<C^{*}(\alpha, \delta)
$$

with $C^{*}$ being defined by (22). Then, the first minimum of the function $f$ is greater than the second one. Analogously for the inverse case we have

$$
C^{*}(\alpha, \delta)<C<C_{\max }(\alpha, \delta)
$$

When the parametric triplet satisfies (34), system (32) supports a homoclinic trajectory that joins the left saddle point with itself. In this case we will call its corresponding solution as pedestal like solution. On the other hand, when (35) is satisfied, the system supports now a homoclinic trajectory that joins the right saddle point with itself and we will call its corresponding solutions as bubble like solution. These subregions of admissible regions are shown in Figure 3 separated by a straight line; the upper one corresponds to bubble type solutions. The cubic surface (22) is the set of bifurcations between "pedestals" and "bubbles" which is a heteroclinic bifurcation of the system (32). We illustrate these bifurcations by showing in Figures 4 to 6 the "evolution" of phase trajectories while the relevant parameters change. 


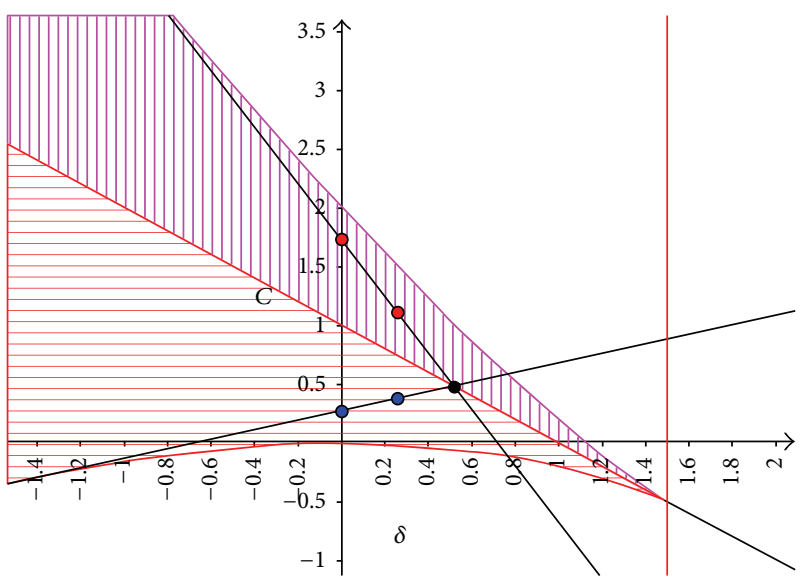

FIgure 3: A slide of the admissible region with $\alpha=-2$. The straight line which divides the dashed regions is the bifurcation set (22). Below this line pedestal like solutions "live" while above this line bubble like solutions exist. The dotted points represent the hysteresis path; see Figures 7 to 11 and the hysteresis curve is represented in Figure 12.

If we restrict ourselves to the parametric region defined by (19), then we observe that the condensate boundary condition value $U_{0}$ is one of the three solutions of the cubic equation

$$
g(x)=-C .
$$

Outside the admissible region, this equation has only one real solution (in the border of the admissible region it has two real solutions). If (10) is accomplished, then (36), for each $U_{0}$, represents a plane in the parametric space $(\alpha \delta C)$ with equation

$$
C=-2 U_{0} \delta-4 U_{0}^{3}-3 \alpha U_{0}^{2}
$$

additionally, it is subjected to the restriction

$$
C_{\min }(\alpha, 0) \leq-4 U_{0}^{3}-3 \alpha U_{0}^{2} \leq C_{\max }(\alpha, 0)
$$

(e.g., if $\alpha<0, C_{\min }(\alpha, 0)=0$ and $\left.C_{\max }(\alpha, 0)=-\alpha^{3} / 4\right)$.

In the example illustrated by Figures 4,5 , and 6 we chose the value for $\alpha=-2$ and the boundary condition $U_{0}=1$. The other parameter values are taken from the straight line

$$
C=-2 \delta+2
$$

that are inside of the admissible region. Bifurcation occurs for $\delta=1$ and $C=0$ as can be seen in Figure 5 and the trio $(\alpha, \delta, C)$ lies in the bifurcation set (22).

4.2. Catastrophe and Hysteresis. Bifurcation theory studies and classifies phenomena characterized by sudden shifts in behavior arising from small changes in circumstances, analyzing how the qualitative nature of equation solutions depends on the parameters that appear in the equation. This may lead to sudden and dramatic changes, for example, the unpredictable timing and magnitude of a landslide. This is the subject matter of catastrophe theory, a branch of

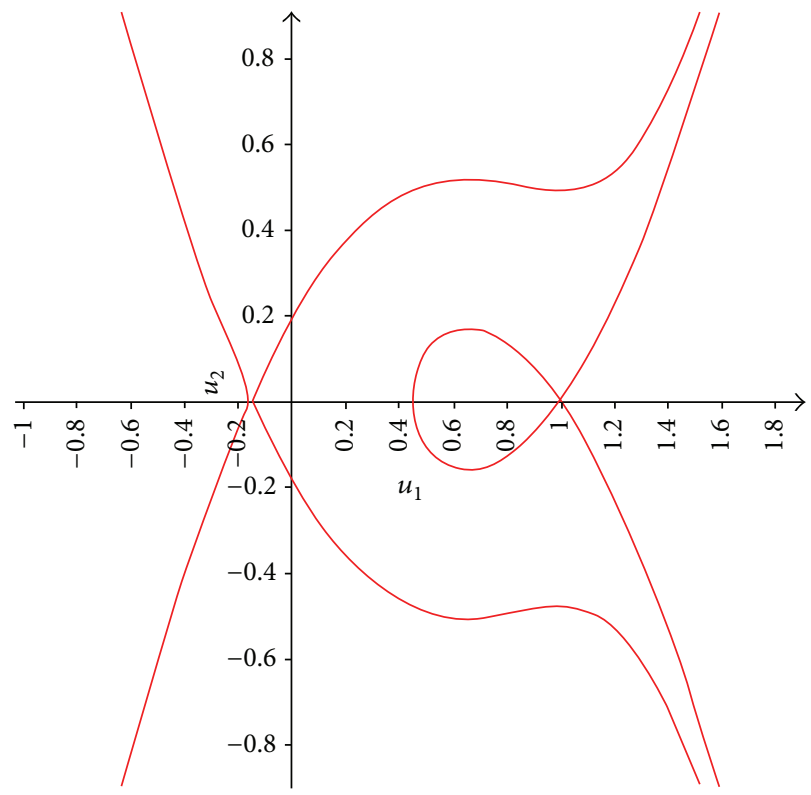

FIgure 4: Bubble phase trajectory, $\delta=0.8, C=0.4$ : the solution is of the bubble type with condensate boundary condition, $U_{0}=1$, located at the saddle on the right.

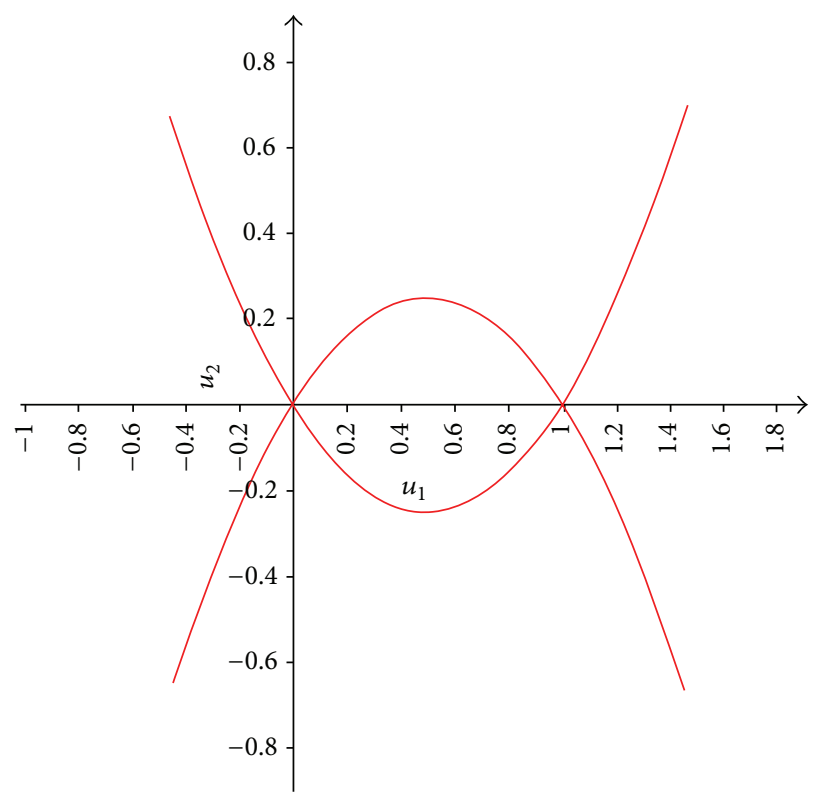

FIGURE 5: Bifurcation: the homoclinic trajectory of the previous figure becomes in two heteroclinic trajectories joining the saddle points. $\delta=1, C=0$.

bifurcation theory, which was originated with the work of the French mathematician René Thom in the 1960s. Catastrophe theory represents the propensity of structural stable systems to display discontinuity behavior. Small changes in certain parameter values of a nonlinear system could cause equilibrium to appear or disappear, or it could change the system state from attracting to repelling one and vice versa. The large and sudden changes in the system behavior 


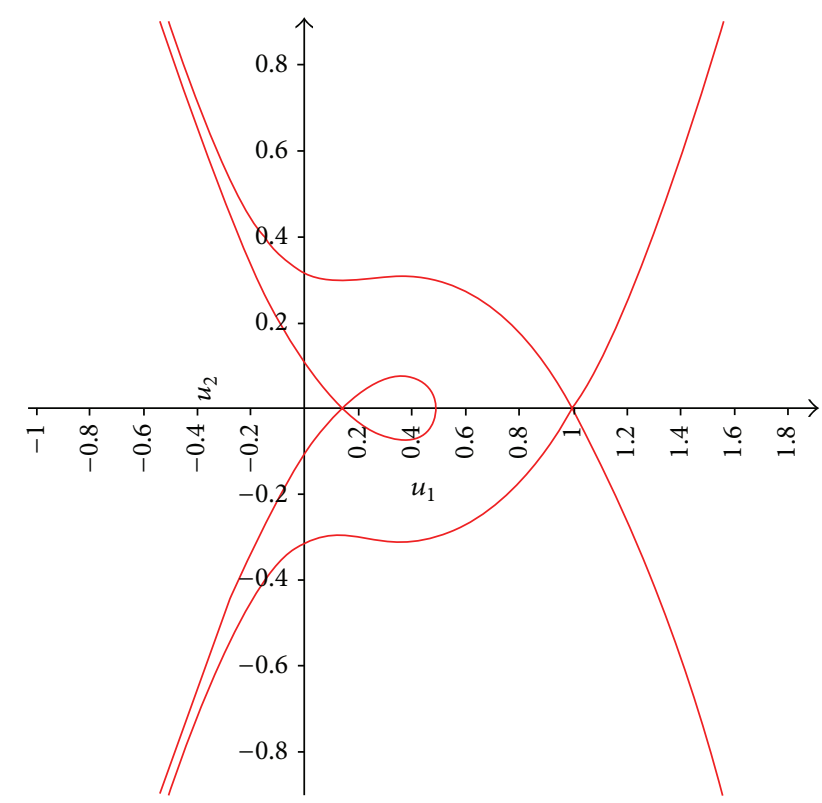

FIgURE 6: A pedestal type solution emerges from different saddle like equilibrium point. In this case we use the condensate boundary condition value $U_{0}=0.12$ and $\delta=1.1, C=-0.2$.

could eventually promote the generation of divergences and hysteresis. Hysteresis as is well known is the tendency of certain materials to conserve one of its properties, in absence of the stimulus that generated it. For extension it is applied to phenomena that not only depend on the actual circumstances but also depend on how these properties have been acquired by the material.

Examined in a larger parameter space, catastrophe theory reveals that such bifurcation points tend to occur as part of well-defined qualitative geometrical structures. In our case, the part of the cubic surface (22) contained in the admissible region is like a catastrophe surface for the parametric family (32) in the following sense: if a parametric trajectory crosses the surface (22), then the system suddenly switches from a bubble type solution to pedestal type one or vice versa (see Figures 7, 8, 9, 10, and 11).

The catastrophic situation takes place in accordance with a hysteresis-like behavior of the family: let us suppose that we begin with traveling waves of certain initial velocity (say $v=c_{0}$ see Figure 7) specifically with a bubble type of solution and the boundary condition $U_{0}$ that will be maintained fixed as the velocity changes (we will preserve the value of the parameter $\alpha$ unchanged). Thus, by modifying the velocity and keeping the boundary condition $U_{0}$ fixed, the parameters $\delta$ and $C$ change according to the lineal relationship (37). Following this trajectory by decreasing the velocity $v$, the surface (22) will be crossed, while the first border condition changes abruptly to a new border condition $\overline{U_{0}}$. At this point the pedestal soliton-like solution suddenly appears. Maintaining now this new border condition and the same solution fixed, we try to return back to the initial state with soliton velocity $\left(v=c_{0}\right)$. But unexpectedly performing these changes the system does not return to

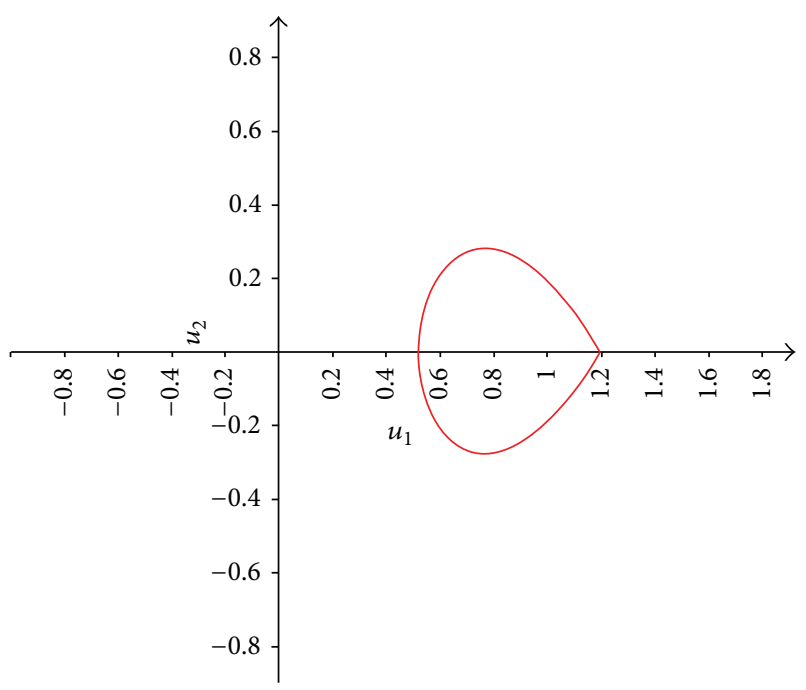

Figure 7: Homoclinic path for a bubble type solution with condensate boundary condition $U_{0}=1.2$; this will be keeping up with the parametric path until bifurcation occurs, $(\delta, C)=(0,1.728)$.

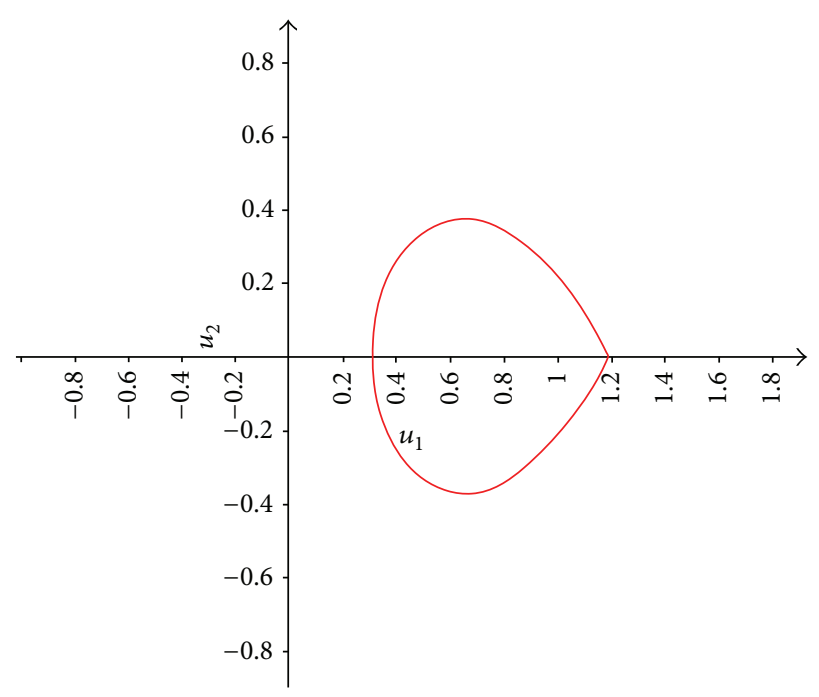

FIGURE 8: Homoclinic path for a bubble solution with condensate boundary condition $U_{0}=1.2$. $(\delta, C)=(0.26,1.104)$.

the initial dynamical behavior; instead the system shows a pedestal type of behavior.

In other words, we start from a bubble type solution and we return to pedestal type solution and vice versa. This behavior in the parameter space is illustrated in Figure 3 with the aid of Figures 7 to 11, where it is shown that the broken dotted straight line that corresponds to hysteresis and the point at the edge represents the catastrophe.

The hysteresis in this model can be observed in the parametric space determined by two parameters: the boundary condition $U_{0}$ and the soliton velocity $v$ as it is depicted in Figure 12. One starts with velocity $v=c_{0}$ and moves keeping up $U_{0}=1.2$ and decreasing $v$ until the critical 


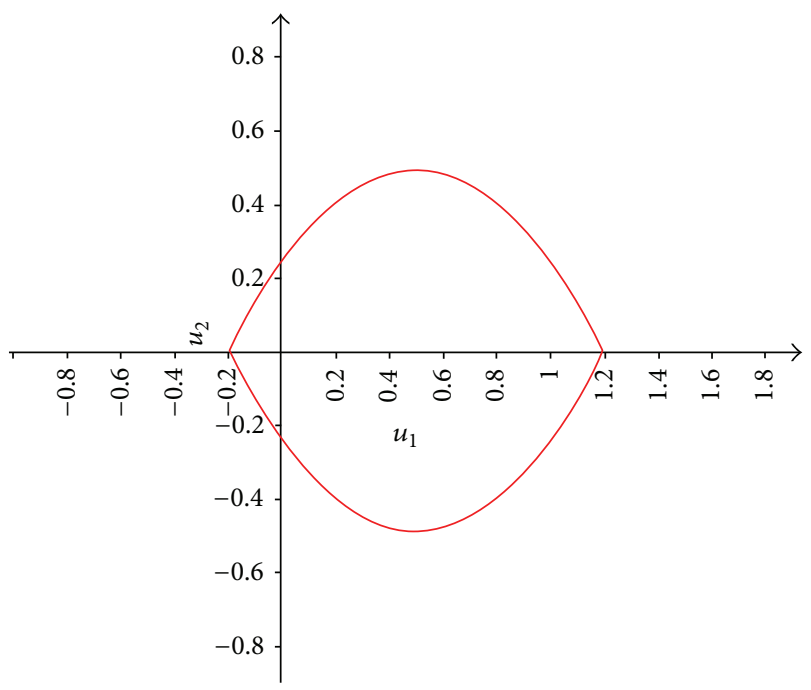

FIGURE 9: Catastrophe switching suddenly from a bubble to a pedestal type of solution. Phase space for the parameter values $(\delta, C)=$ $(0.52,0.48)$ showing heteroclinic trajectories joining the actual condensate boundary condition $U_{0}=1.2$ with an emerging boundary condition $U_{0}=-0.2$.

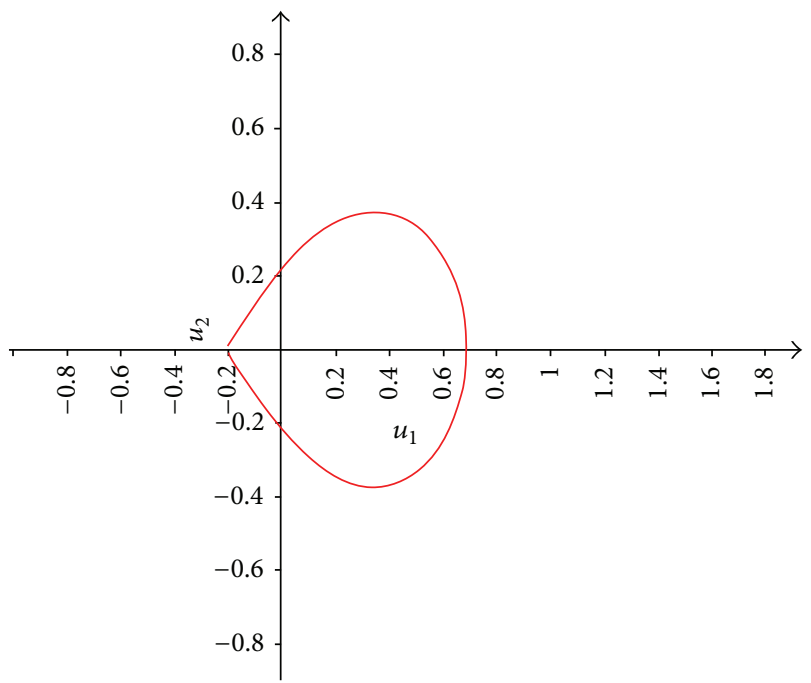

FIgURE 10: Homoclinic trajectory for a pedestal type solution with condensate boundary condition $U_{0}=-0.2 .(\delta, C)=(0.26,0.376)$.

value of $c_{1}=\sqrt{c_{0}^{2}-\delta_{0} \beta}$ is achieved while the condensate boundary condition switches suddenly to $U_{0}=-0.2$. Next, one maintains this boundary condition fixed and the velocity $v$ returns to its initial value $c_{0}$. The parameter $\delta_{0}$ is the critical value when the system has two heteroclinical trajectories (see Figure 9). We can observe that the velocity of the nonlinear structures is restricted by the boundary conditions and the constant of integrations $C$ that also depends on the other parameters of equation of motion (2). Eventually the switching between bubble and pedestal states of the system occurs without releasing or absorbing energy in absence of other types of forces. Thus, the energy necessary for appearing

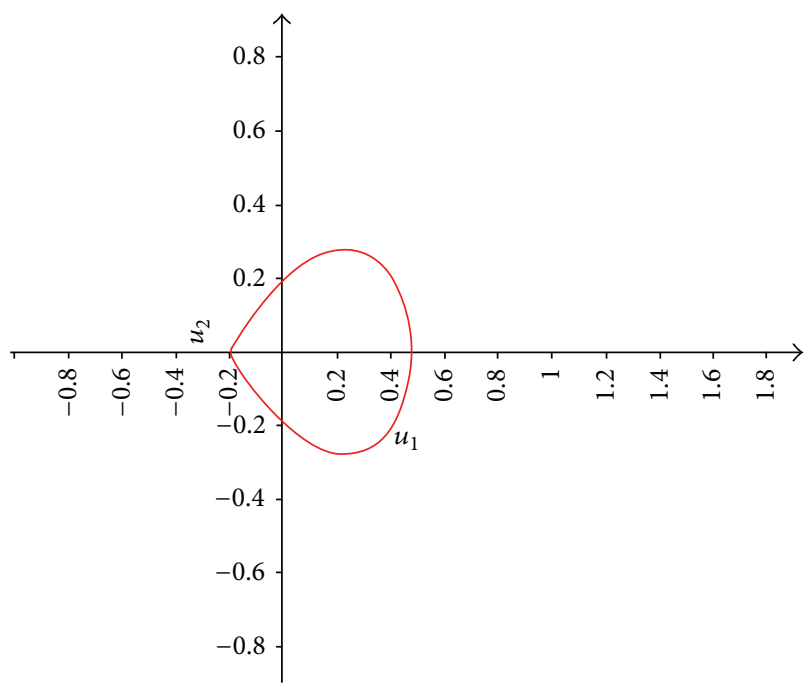

FIGURE 11: Homoclinic trajectory for a pedestal type solution with condensate boundary condition $U_{0}=-0.2,(\delta, C)=(0,0.272)$.

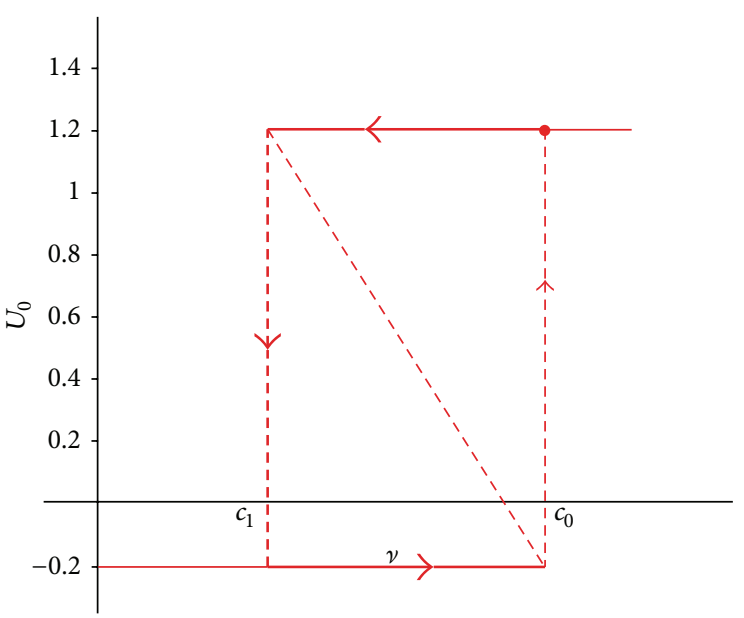

FIGURE 12: Hysteresis of condensate boundary condition $U_{0}$ as function of the traveling wave velocity $v$. Starting with $v=c_{0}$, keeping up $u=U_{0}=1.2$, and decreasing $v$ until the critical value $c_{1}=\sqrt{c_{0}^{2}-\delta_{0} \beta}$ is achieved, the condensate boundary condition switches suddenly to $U_{0}=-0.2$ maintaining it fixed while $v$ returns to the initial value $c_{0}$. Other parameter values were chosen as $\alpha=-2, c_{0}=2, \beta=0.173$, and $\delta_{0}=0.52$.

bubble excitations should be equal to the energy of pedestal excitations. This conjecture subsequently will provide us with the new restriction for determining the relationship between the soliton velocities in dependence on the main parameters of the motion equation. As we can observe, it is supposed that in the gel or "solid" state the pedestal excitations could emerge, while in the liquid sate bubble perturbations should propagate along the nerve. This behavior could tell us that inside the membrane the second type of phase transition occurs, when the boundary conditions for the soliton-like solutions in the model equation are of condensate type $U_{0}$. As usual we can consider second order phase transition only 
those of the transitions in which below and above the critical point only one phase lives in each sector.

\section{Conclusions}

We have discussed in this work the hysteresis and catastrophe phenomena that appear in the parameter space when nontopological type of solitons, that is, bubble and pedestal solitons, emerges from background along the axon. These finding were done on the basis of the pioneering model developed by Heinburg and Jackson [11]. These solutions propagate over the spatially homogeneous background. It is interesting to note that the appearance of solitons does not manifest as a Markovian process; instead the propagation of pulses apparently occurs with memory. The hysteresis curve in dependence on the parameter $v$, the traveling wave velocity, is depicted in Figure 12. The appearance of catastrophe and hysteresis suggests for us that if the system initially supports a bubble structure on the condensate, then, after parameter variations, the condensate changes to another value while the bubble solution is transformed into pedestal soliton lying on different background or condensate with slightly minor value.

Both solutions, the bubble and the soliton (pedestal), on the background obtained as particular soliton-like solutions for specific values of parameters, could be used by the nerve for enhancing confidentiality in communication tasks. For instance, as the bubble soliton amplitude vanishes or minimizes during propagation along the nerve, this wave could be used to perform communication transmission for security, whereas the required information can be retrieved by the dark/bright soliton conversion on the background. Apparently, these solutions could conform some informational code structures for preserving and transmitting valid information along the nerve. In this case we can observe that the hysteresis could be considered as a natural filter mechanism for securely transmitting signals along the nerve system.

\section{Conflict of Interests}

The authors declare that there is no conflict of interests regarding the publication of this paper.

\section{Acknowledgments}

Maximo A. Agüero is indebted to Professor V. G. Makhankov for constant support and discussions. This work was supported in part by the Secretary of Education of Mexico under the Project PROMEP 103.5/13/9347 for developing research scientific groups.

\section{References}

[1] A. L. Hodgkin and A. F. Huxley, "A quantitative description of membrane current and its application to conduction and excitation in nerve," The Journal of Physiology, vol. 117, no. 4, pp. 500-544, 1952.

[2] R. Fitzhugh, "Impulses and physiological states in theoretical models," Biophysical Journal, vol. 1, pp. 445-466, 1961.
[3] J. Nagumo, S. Arimoto, and S. Yoshizawa, "An active pulse transmission line simulating nerve axon," Proceedings of the IRE, vol. 50, no. 10, pp. 2061-2070, 1962.

[4] F. Ongay and M. Agero, "Bifurcaciones del sistema Fitzhugh Nagumo," Ciencia Ergo Sum, vol. 17, no. 3, pp. 295-306, 2011.

[5] B. Katz, Nerve, Muscle, and Synapse, McGraw-Hill, New York, NY, USA, 1966.

[6] C. B. Muratov, "A quantitative approximation scheme for the traveling wave solutions in the Hodgkin-Huxley model," Biophysical Journal, vol. 79, no. 6, pp. 2893-2901, 2000.

[7] I. Tasaki and P. M. Byrne, "Heat production associated with a propagated impulse in bullfrog myelinated nerve fibers," Japanese Journal of Physiology, vol. 42, no. 5, pp. 805-813, 1992.

[8] M. Wu, B. A. Kalinikos, and C. E. Patton, "Generation of dark and bright spin wave envelope soliton trains through selfmodulational instability in magnetic films," Physical Review Letters, vol. 93, no. 15, Article ID 157207, 2004.

[9] N. N. Rosanov, N. V. Vysotina, A. N. Shatsev, I. V. Shadrivov, and Y. S. Kivshar, "Hysteresis of switching waves and dissipative solitons in nonlinear magnetic metamaterials," JETP Letters, vol. 93, no. 12, pp. 743-746, 2011.

[10] E. Wilke, "Das Problem der Reizleitung im Nerven vom Standpunkte der Wellenlehre aus betrachtet," Pflüger's Archiv für die gesamte Physiologie des Menschen und der Tiere, vol. 144, no. 1-2, pp. 35-38, 1912.

[11] T. Heinburg and A. Jackson, "On soliton propagation in biomembranes and nerves," Proceedings of the National Academy of Sciences of the United States of America, vol. 102, no. 28, pp. 9790-9795, 2005.

[12] B. Lautrup, R. Appali, A. D. Jackson, and T. Heimburg, "The stability of solitons in biomembranes and nerves," European Physical Journal E, vol. 34, no. 6, pp. 1-9, 2011.

[13] E. Vargas, U. van Rienen, and T. Heimburg, "Periodic solutions and refractory periods in the soliton theory for nerves and the locust femoral nerve," Biophysical Chemistry, vol. 153, no. 2-3, pp. 159-167, 2011.

[14] H. Barz, A. Schreiber, and U. Barz, "Impulses and pressure waves cause excitement and conduction in the nervous system," Medical Hypotheses, vol. 81, no. 5, pp. 768-772, 2013.

[15] F. Contreras, H. Cervantes, M. Aguero, and M. de Lourdes Najera, "Classic and non-classic soliton like structures for traveling nerve pulses," International Journal of Modern Nonlinear Theory and Application, vol. 2, no. 1, pp. 7-13, 2013.

[16] R. Appali, U. van Rienen, and T. Heimburg, "A comparison of the hodgkin-huxley model and the soliton theory for the action potential in nerves," Advances in Planar Lipid Bilayers and Liposomes, vol. 16, pp. 275-299, 2012.

[17] F. Contreras, O. Pavon, F. Ongay, and M. Aguero, "Non topologicalal solitons as traveling pulses along the nerve," International Journal of Modern Nonlinear Theory and Application, vol. 2, pp. 195-200, 2013. 

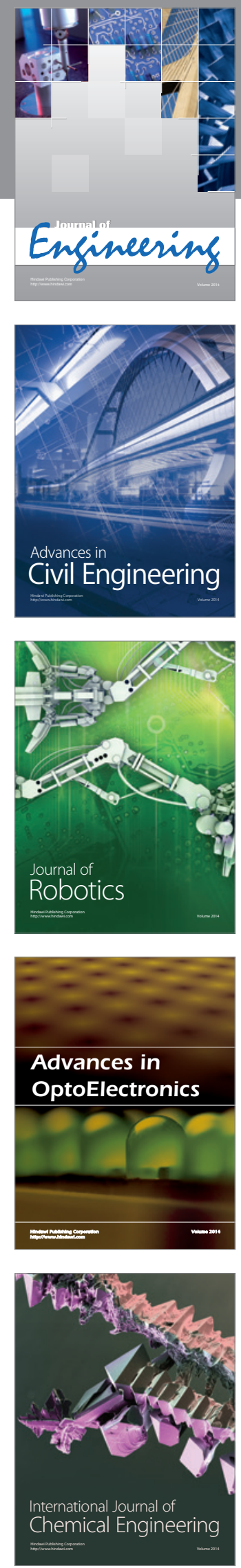

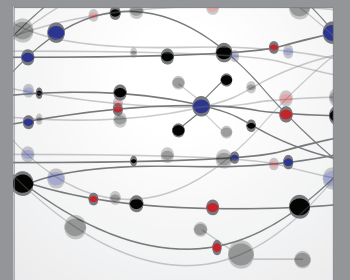

The Scientific World Journal
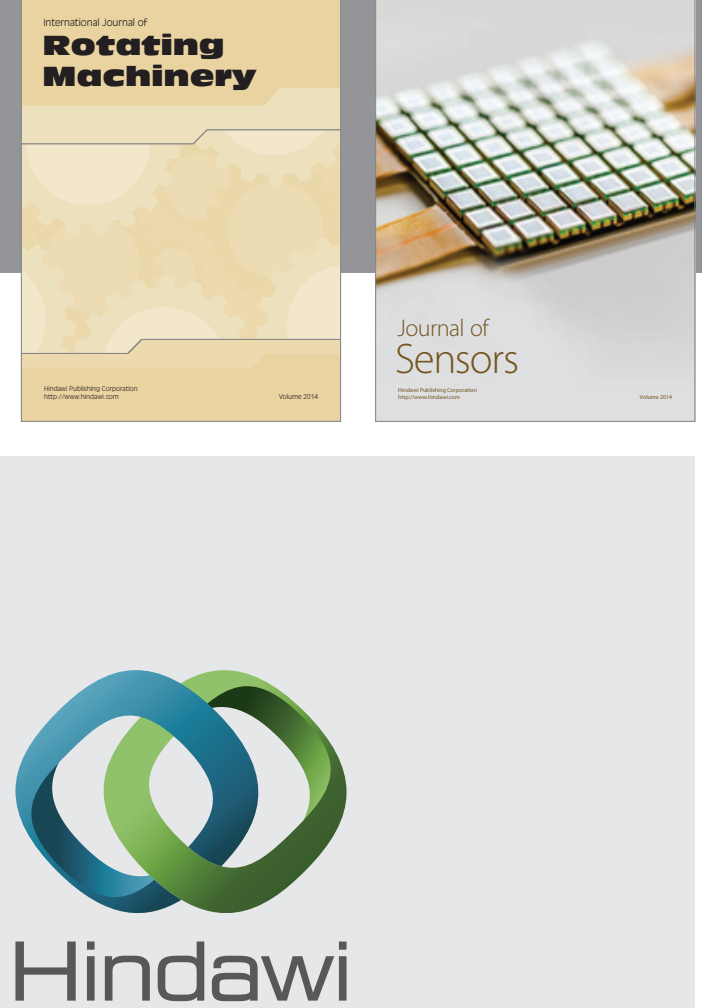

Submit your manuscripts at http://www.hindawi.com
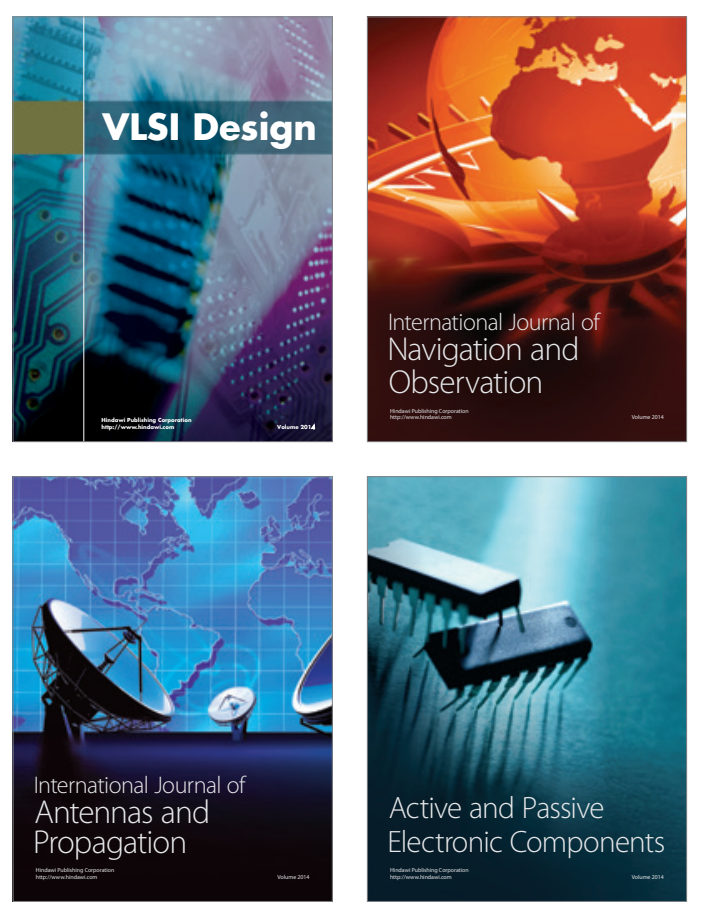
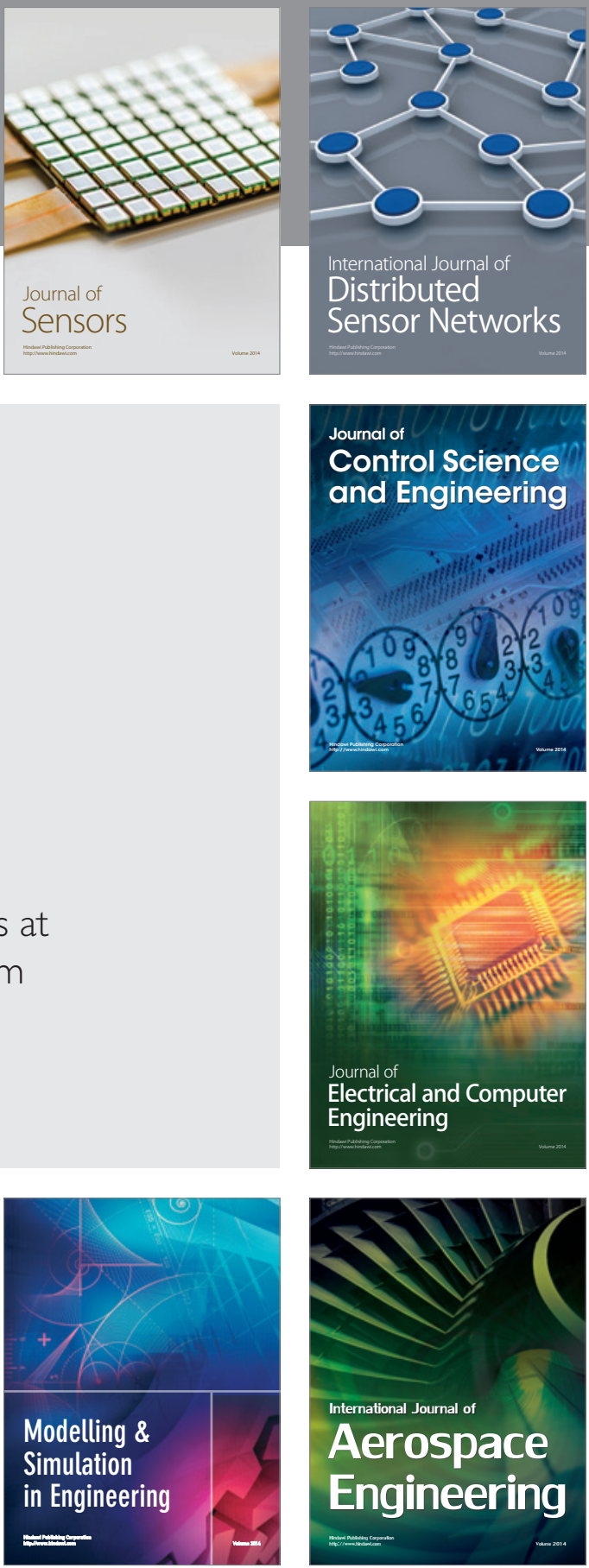

Journal of

Control Science

and Engineering
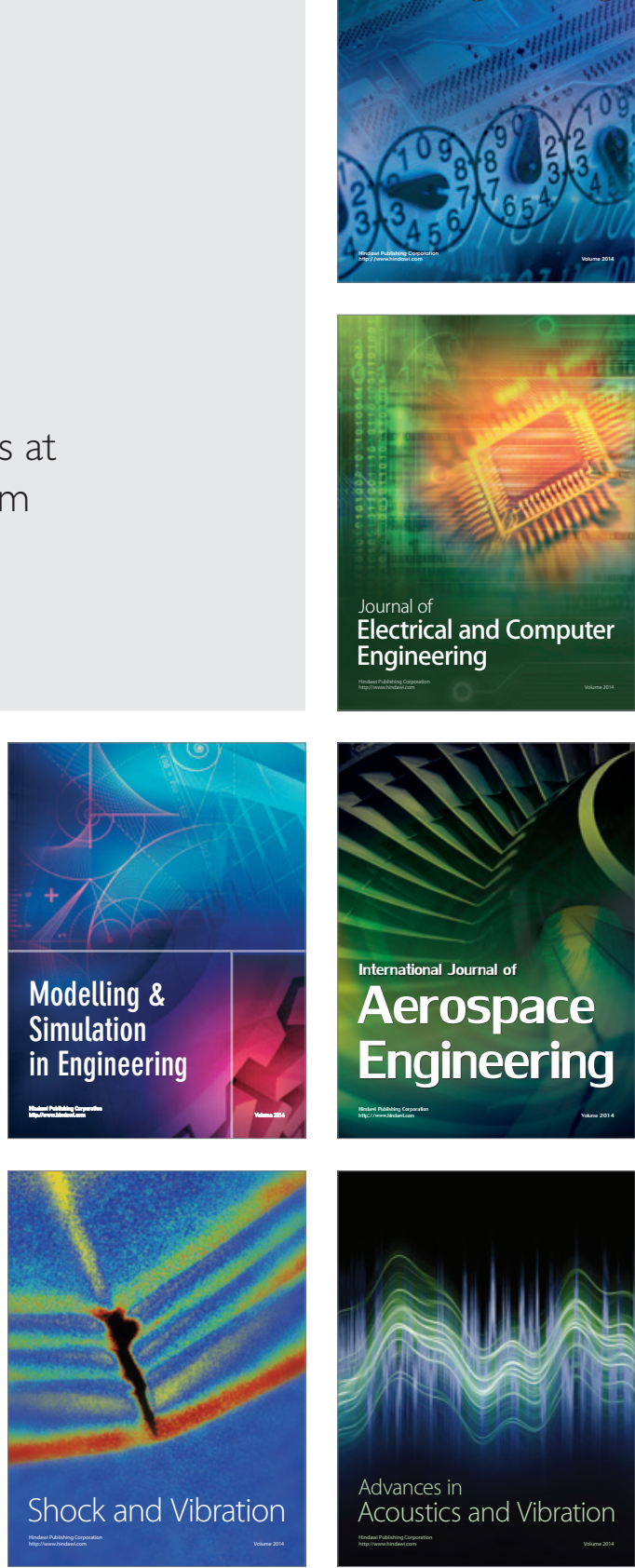